\title{
Vegetación acuática y estado trófico de las lagunas andinas de San Pablo y Yahuarcocha, provincia de Imbabura, Ecuador
}

\author{
Esteban Terneus Jácome ${ }^{1}$ \\ ${ }^{1}$ Escuela de Biología Aplicada, Universidad Internacional del Ecuador, Quito, Ecuador \\ hterneus@internacional.edu.ec \\ Recibido: 2014-05-29; aceptado: 2014-11-17
}

\section{RESUMEN.-}

San Pablo y Yahuarcocha son dos de las cuatro lagunas que se encuentran entre 2200 y $2800 \mathrm{~m}$ de altitud en Ecuador. Al estar localizadas en este rango altitudinal, son más productivas que las de la zona de páramo, por la elevada concentración de nutrientes en sus aguas, producto de la influencia humana en sus áreas circundantes. El presente estudio analiza el estado trófico de las lagunas de San Pablo y Yahuarcocha y su repercusión en la estratificación de la vegetación acuática y en su composición florística.

Se registraron veintiún especies de plantas acuáticas en San Pablo y nueve en Yahuarcocha; cinco especies se encuentran en ambas lagunas incluyendo la "Totora" (Scirpus californicus (C.A. Mey.) Steud), utilizada en la elaboración de esteras. En San Pablo, una de las especies más importantes es Ceratophyllum demersum Hutch.\& Dalziel, 1930 única especie registrada para el Ecuador en lagunas andinas y que crece hasta los siete metros de profundidad. Esta especie sirve como alimento para el Bass (Morone saxatilis Walbaum, 1792), el principal recurso en la pesca artesanal de los indígenas de la zona y que también se encuentra presente únicamente en esta laguna. En Yahuarcocha, Potamogeton pusillus L., crece hasta los $3.10 \mathrm{~m}$ de profundidad y también constituye una fuente de recursos alimentarios para peces como la Tilapia (Tilapia Mozambique (Peters, 1852) y los Guppis (Poecilia reticulata W. K. H. Peters, 1859).

Yahuarcocha se puede catalogar como una laguna eutrófica, debido a que su alcalinidad fue de $956 \mathrm{mg} / \mathrm{l}$, y su conductividad fue superior a $620 \mathrm{~ms}$; la transparencia del agua fue menor a dos metros de profundidad y estuvo dominada por una sola especie (Potamogeton pusillus). En cambio, San Pablo es un lago mesotrófico, con una conductividad menor a $320 \mathrm{~ms}$ y una alcalinidad de $226 \mathrm{mg} / \mathrm{l}$, transparencia de agua sobre los $3 \mathrm{~m}$ de profundidad y presencia de al menos veinte especies de plantas acuáticas que originan hábitats heterogéneos, óptimos para el establecimiento de comunidades mixtas de plantas alrededor de toda la laguna.

PALABRAS CLAVES: estratificación, estado trófico, macronutrientes, transparencia, vegetación acuática

\section{ABSTRACT.-}

San Pablo and Yahuarcocha are two of the four lakes which are located in Ecuador in a range of 2200 to $2800 \mathrm{~m}$ of altitude. Their location within this altitude range qualified them as more productive than those lakes from the Páramo. This study analyses the trophic state of San Pablo and Yahuarcocha lakes and how it influences in the stratification of the vegetation and the floristic composition. In total, twenty one species of aquatic macrophytes were found in San Pablo and nine in Yahuarcocha; five species were growing in both places, including the "Totora" (Scirpus californicus (C.A. Mey.) 
Steud.), which is used in the production of mats. One of the most important species in San Pablo is Ceratophyllum demersum Hutch.\& Dalziel, 1930., the only specie recorded for the Andes of Ecuador, which grows up to seven meters depth. It has a great nutritious value for the Bass (Morone saxatilis Walbaum, 1792), important fish for the indigenous diet. On the other hand, Potamogeton pusillus L. is growing until $3.10 \mathrm{~m}$ depth in Yahuarcocha and it is also a good food resource for fishes like Tilapia (Tilapia Mozambique (Peters, 1852) and the Guppis (Poecilia reticulata W. K. H. Peters, 1859). Yahuarcocha could be catalogued as an eutrophic lake. The alkalinity was $956 \mathrm{mg} / \mathrm{l}$, conductivity was over $620 \mathrm{~ms}$, water transparency was less than two meters depth and there was only one dominating specie (Potamogeton pusillus). Meanwhile, San Pablo is a mesotrophic lake. The conductivity was less than $320 \mathrm{~ms}$, the alkalinity was $226 \mathrm{mg} / \mathrm{l}$, water transparency was above $3 \mathrm{~m}$ in depth and there were at least twenty species of aquatic plants, which arises heterogeneous habitats with the best conditions for the mixed communities establishments around the lake.

KEYWORDS.- aquatic vegetation, macronutrients, stratification, transparency, trophic state.

\section{INTRODUCCIÓN}

La presencia de al menos 4000 viviendas alrededor delas lagunas San Pablo y Yahuarcocha (CEPCU, 1999) contribuyen a su deterioro ambiental provocando descensos en los niveles naturales de sus cuerpos de agua, contaminación por eliminación de aguas servidas, incremento de la turbidez del agua y contaminación de afluentes por pesticidas provenientes de actividades agrícolas y ganaderas. Todos estos elementos influyen significativamente en la distribución y el establecimiento de las comunidades de plantas acuáticas (Mallin, 2000), y han sido los causantes de los cambios en la estructura de muchos ecosistemas acuáticos como el lago de La Herrera en Colombia (Wijninga et al., 1989; Cortés et al., 1980). En el lago mexicano de Cuitzeo, Rojas y Novelo (1995) demostraron que la disminución en el nivel de agua, de aproximadamente $1.35 \mathrm{~m}$ en un período de veinticinco años, ha impactado sobre las comunidades de plantas acuáticas que crecen en el lago. Un fenómeno similar podría estar ocurriendo en San Pablo y Yahuarcocha, producto de la influencia humana y el uso excesivo de los recursos en los alrededores de las lagunas. Para recuperarlas y mantenerlas en buen estado es necesario conocer su funcionamiento y la concentración de carga orgánica en sus aguas.
En Ecuador el estudio realizado por Terneus (2002) hace referencia a la distribución de las plantas acuáticas en setenta lagunas altoandinas del Ecuador, aportando la base fundamental para determinar los patrones de distribución horizontal de estas plantas a lo largo de una gradiente de profundidad y la distribución geográfica de algo más de sesenta especies a lo largo de los sistemas lacustres de la cordillera de Los Andes ecuatorianos. En el callejón interandino existen un estudio realizado por Gunkel (2000) en el Lago San Pablo y Cuicocha, en donde se pone en evidencia la importancia de los nutrientes en el sustrato sobre la composición y estructura de las plantas acuáticas. Sin embargo, no existen estudios relacionados con el efecto de la química de agua sobre los patrones de distribución de la vegetación acuática y el estado trófico de las lagunas de San Pablo y Yahuarcocha, esta última considerada también como una de las lagunas más importantes del callejón interandino del Ecuador por su historia, turismo y tradición.

En este contexto, los objetivos de este estudio son:1) Determinar el estado trófico de las lagunas de San Pablo y Yahuarcocha, a través del análisis de parámetros biológicos, físicos y químicos, y 2) Analizar si la diversidad de plantas acuáticas y su biomasa están relacionadas con el estado trófico de las lagunas. 


\section{MATERIALES Y MÉTODOS}

\section{Área de estudio.-}

Las dos lagunas se encuentran localizadas en la provincia de Imbabura y por su altitud geográfica ocupan una zona intermedia entre el valle del Chota y las zonas altas del callejón interandino (Figura 1). Junto a las lagunas del Cunrro y Yambo son las únicas lagunas de Ecuador ubicadas en rangos altitudinales comprendidos entre 2200 y $2800 \mathrm{~m}$ de altitud (Terneus, 2002). San Pablo y Yahuarcocha difieren en su dinámica por la altitud geográfica, superficie y profundidad de agua (Tabla 1) La presencia de asentamientos humanos en sus alrededores y su entorno paisajístico las diferencia de las lagunas que se encuentran en zonas de páramo.

Tabla 1. Características geofísicas y ubicación de las lagunas San Pablo y Yahuarcocha

\begin{tabular}{lll}
\hline Laguna & San Pablo & Yahuarcocha \\
\hline Ubicación & Faldas del volcán Imbabura & Faldas del Cerro El Redondo \\
Coordenadas & $00^{\circ} 13^{\prime} \mathrm{N} ; 78^{\circ} 14^{\prime} \mathrm{W}$ & $00^{\circ} 22^{\prime} \mathrm{N} ; 78^{\circ} 6^{\prime} \mathrm{W}$ \\
Altitud & $2650 \mathrm{~m}$ & $2200 \mathrm{~m}$ \\
Superficie & $583 \mathrm{ha}$ & $230 \mathrm{ha}$ \\
Profundidad máxima & $30 \mathrm{~m}$ & $7 \mathrm{~m}$ \\
Afluentes & Ríos Itambi y Manantial & Quebrada Manzana Huaycu \\
\hline
\end{tabular}
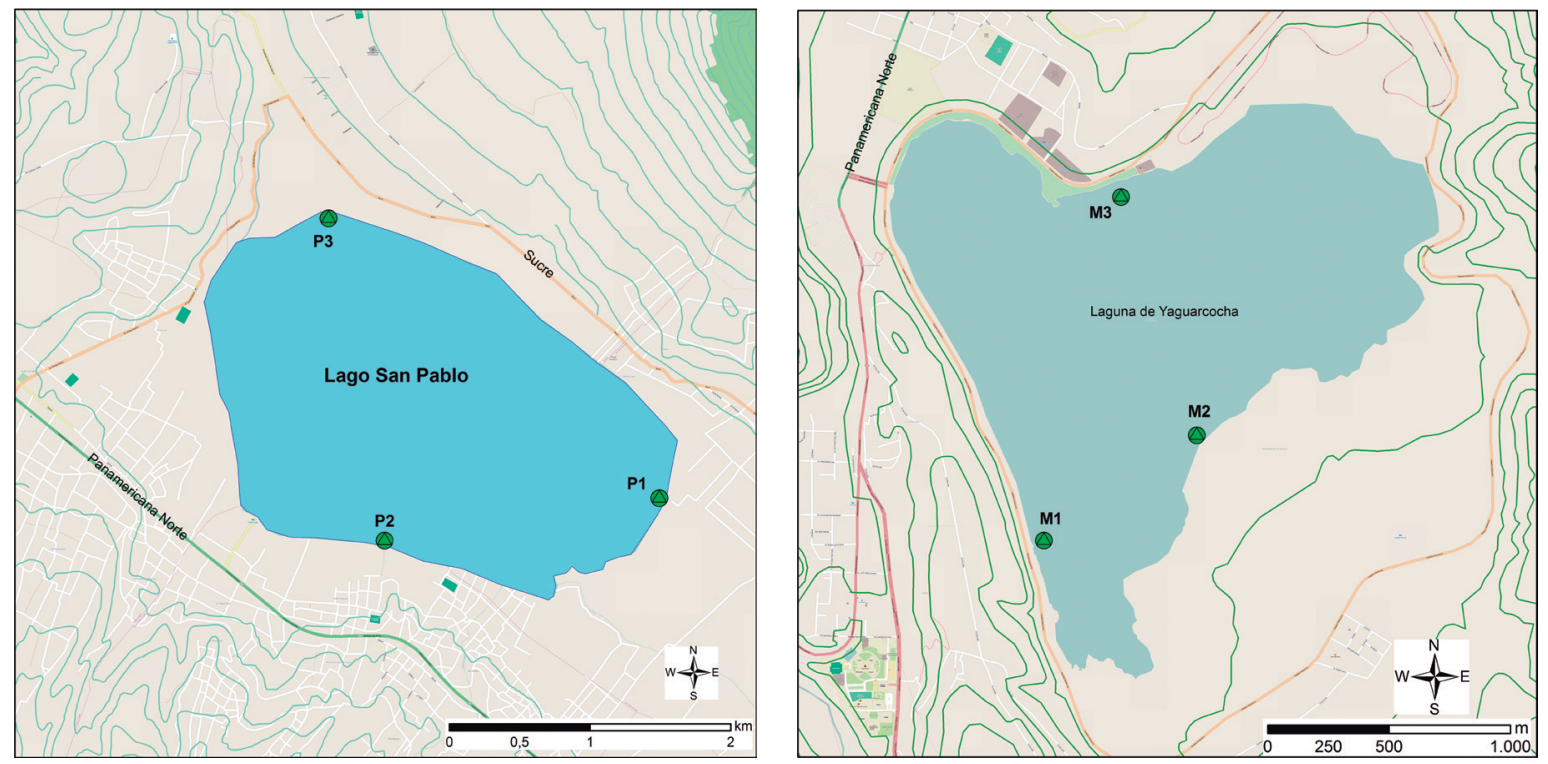

Figura 1. Imagen satelital de las dos lagunas de estudio, San Pablo a la izquierda y Yahuarcocha a la derecha. Los puntos de muestreo se exponen en color rojo, lugares donde se establecieron los transectos.

Junto al lago San Pablo se asientan las parroquias rurales de San Pablo, Eugenio Espejo, González Suárez y San Rafael, con una población total de aproximadamente 20000 habitantes.
La principal actividad en la zona es la agricultura $(30 \%)$, seguida por la industria manufacturera (25\%) y otras (Kiersch et al., 2004; Carrera de la Torre, 1990). 
En Yahuarcocha se asientan los barrios de Aluburo, Yahuarcocha y Priorato con una población de aproximadamente 3000 habitantes. Las principales actividades son la agricultura y la pesca artesanal (Mueses, 2010; Carrera de la Torre, 1990).

\section{Análisis del agua y de su vegetación.-}

El estado trófico de las lagunas se evaluó en base a las características fisicoquímicas del agua como transparencia, conductividad eléctrica, alcalinidad, $\mathrm{pH}$, temperatura y concentración de macronutrientes (Roldán y Ramírez, 2008; Wijninga et al., 1989). La transparencia del agua fue medida con un disco Secchi, el mismo que se introdujo en el agua progresivamente, a varios metros de profundidad, hasta que la visibilidad permita identificarlo. La conductividad eléctrica se midió con un conductivímetro electrónico (Hanna HI 8633). El pH y la temperatura se midieron con un dispositivo electrónico (Jenway 3150) y la alcalinidad se midió por titulación mediante una solución de $\mathrm{HCl}(0.1 \mathrm{~N})$ en los laboratorios de la Universidad Internacional del Ecuador. La concentración de macronutrientes ( $\mathrm{Ca}, \mathrm{Mg}, \mathrm{Na}, \mathrm{Fe}, \mathrm{Pb}, \mathrm{Al}, \mathrm{K}, \mathrm{SO}_{4}$ ) se analizó en los laboratorios de la Universidad de Plymouth, Inglaterra. Se utilizó un espectrofotómetro de absorción atómica (Terneus, 2001).

Los datos florísticos y ambientales fueron tomados a lo largo de transectos de profundidad ubicados desde la orilla hacia el centro de la laguna y en diferentes puntos de la misma. Estos datos fueron tomados a diferentes profundidades y a intervalos de $1 \mathrm{~m}$ de largo, considerando el límite máximo de profundidad al cual crecen las plantas acuáticas en cada transecto (Terneus, 2002). La vegetación fue dividida en tres estratos: 1. Superficial: compuesto por especies cuyo follaje mantiene contacto con la atmósfera; 2. Medio: especies que crecen desde $40 \mathrm{~cm}$ de profundidad en adelante; 3. Profundo: especies que crecen exclusivamente a más de un metro de profundidad. Adicionalmente, las especies fueron clasificadas, según su forma de vida, en emergentes, sumergidas y flotantes. Todas las plantas acuáticas fueron colectadas con un trinche desde un bote. Las muestras fueron procesadas y depositadas en el Herbario QCA de la Pontificia Universidad Católica del Ecuador (PUCE) y de la Universidad de Aarhus, Dinamarca (AAU).

\section{Análisis estadístico.-}

Los datos físico-químicos fueron correlacionados entre sí, utilizando el índice de Spearman, adecuado para variables con valores heterogéneos y que no se ajustan a una distribución normal (Norman y Streiner 1998; Kent y Coker 1992). Este índice mide la correspondencia entre rangos y, por tanto, no es una medida de correlación lineal (Kent y Coker 1992). Se utilizó la prueba de significación de Wilcoxon, la cual se aplica sobre la base de signos y rangos, considerando la magnitud de las diferencias entre los rangos de los pares de datos de las muestras analizadas (Norman y Streiner 1998; Kent y Coker 1992). Con la prueba de Wilcoxon se determinó si existen diferencias significativas en las concentraciones de macronutrientes entre las dos lagunas muestreadas.

\section{RESULTADOS}

\section{Composición florística.-}

Se encontraron 25 especies de plantas acuáticas en las dos lagunas, 21 en San Pablo y nueve en Yahuarcocha; solamente cinco especies, en su mayoría flotantes, se encontraron en las dos lagunas. Catorce especies fueron monocotiledóneas, diez dicotiledóneas y un helecho acuático (Figura 2). La forma de vida más diversa fue la de las plantas acuáticas emergentes $(36 \%)$, seguidas por las flotantes y sumergidas, las dos con igual riqueza de especies $(32 \%)$ (Tabla 2). 

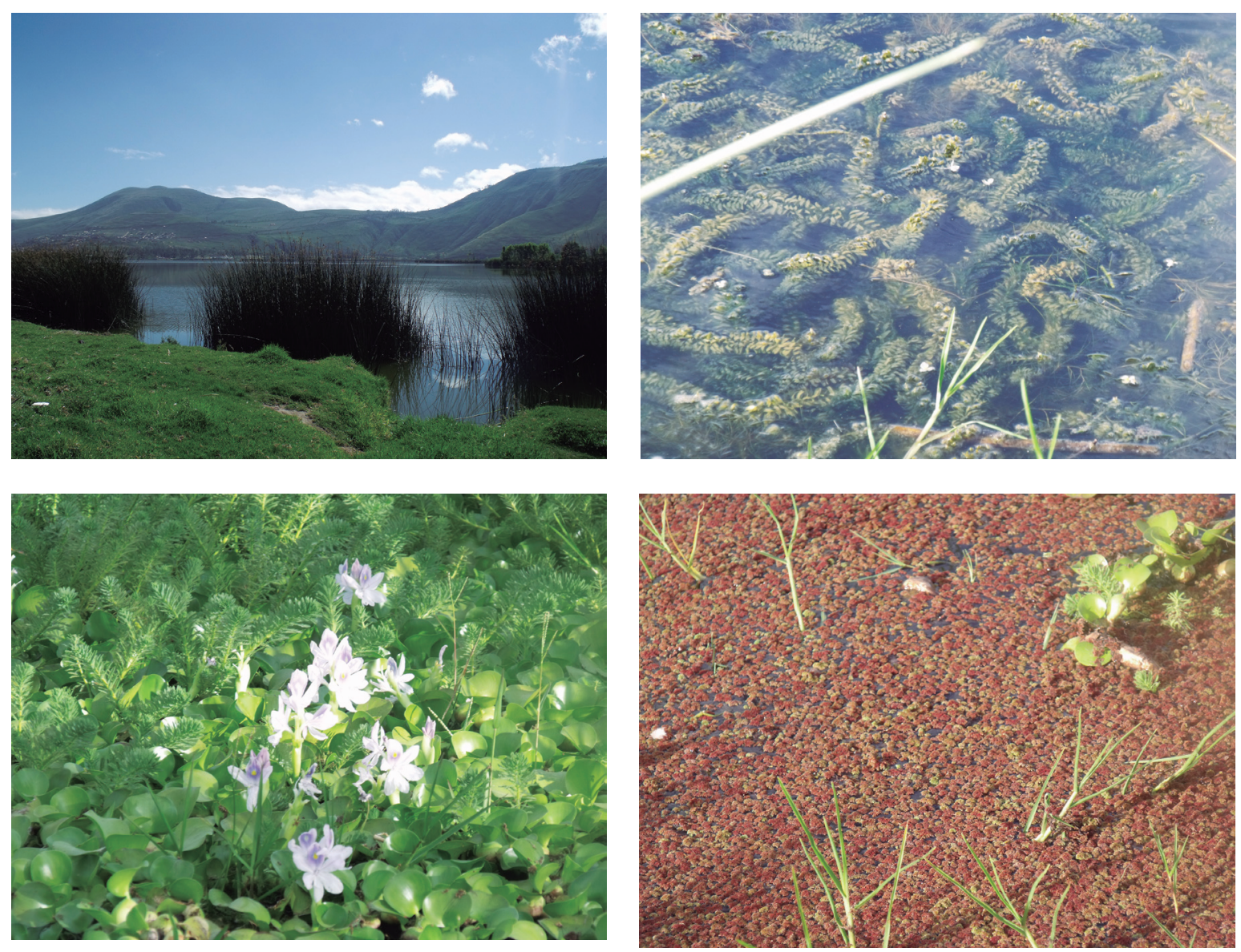

Figura 2. Especies de plantas acuáticas compartidas entre las lagunas de San Pablo y Yahuarcocha: Scirpus californicus, Elodea canadensis, Eichhornia crassipes y Azolla filiculoides.

En la laguna San Pablo el agua es transparente hasta $3.5 \mathrm{~m}$ de profundidad y en Yahuarcocha hasta $1.6 \mathrm{~m}$. La especie dominante a siete metros de profundidad máxima de colonización en San Pablo fue Ceratophyllum demersum. En Yahuarcocha, Potamogeton pusillus fue la especie encontrada a mayor profundidad $(3.10 \mathrm{~m})$. Esta especie es muy abundante entre $0.10 \mathrm{y}$ dos metros de profundidad (Tabla 2).

En San Pablo, el estrato superficial está representado por Scirpus californicus y algunas especies de Lemnaceae, en el estrato medio está Myriophyllum quitense y Elodea canadensis como las especies más abundantes, y en el estrato profundo se encuentran Ceratophyllum demersum y Potamogeton pectinatus (Tabla 2). En Yahuarcocha el estrato superficial está dominado por Myrio- phyllum aquaticum, Scirpus californicus y Typha domingensis, mientras que los estratos medio y el profundo están dominados por Elodea canadensis y Potamogeton pusillus respectivamente (Tabla 2).

\section{Factores ambientales.-}

Los factores ambientales difieren significativamente entre las dos lagunas. Esto es evidente si se examinan las altas concentraciones de macronutrientes ( $\mathrm{Ca}, \mathrm{Na}, \mathrm{Fe}, \mathrm{Mg}$ y K ) y la presencia de plomo en Yahuarcocha (Tabla 3). La prueba de rangos de Wilcoxon indica que las diferencias fueron significativas en la concentración de macronutrientes entre las lagunas de estudio $(\mathrm{W}+17.5$ y W-10.5), valores que se encuentran dentro de los rangos críticos de Wilcoxon (2-26) a un nivel de significación de 0.05 . 
Tabla 2. Tipos de vegetación, especies compartidas, límite de distribución y estratificación de las plantas acuáticas en las lagunas San Pablo y Yahuarcocha

\begin{tabular}{|c|c|c|c|}
\hline Especies & $\begin{array}{l}\text { Rango de } \\
\text { profundidad (m) }\end{array}$ & Forma de vida & Estrato \\
\hline \multicolumn{4}{|l|}{ Especies de San Pablo } \\
\hline Wolffia brasiliensis Wedd. & $0,00-0,00$ & Flotante & Superficial \\
\hline Wolffiela oblonga (Phil.) Hegelm & $0,00-0,00$ & Flotante & Superficial \\
\hline Spirodela intermedia W. Koch & $0,00-0,00$ & Flotante & Superficial \\
\hline Azolla filiculoides Lam* & $0,00-0,00$ & Flotante & Superficial \\
\hline Eichhornia crassipes (Mart.) Solms.* & $0,00-0,00$ & Flotante & Superficial \\
\hline Lemna gibba L. & $0,00-0,00$ & Flotante & Superficial \\
\hline Lemna minuta Kunth* & $0,00-0,00$ & Flotante & Superficial \\
\hline Polygonum hydropiperoides Michx & $0,01-0,10$ & Emergente & Superficial \\
\hline Bidens laevis (L.) B.S.P. & $0,01-0,10$ & Emergente & Superficial \\
\hline Ludwigia inclinata (L.f.) M. Gómez & $0,01-0,15$ & Emergente & Superficial \\
\hline Hydrocotyle umbellata $\mathrm{L}$. & $0,01-0,20$ & Emergente & Superficial \\
\hline Cardamine bonariensis Pers. & $0,01-0,40$ & Emergente & Superficial \\
\hline Phragmites australis (Cav.) Trin. Ex Steud & $0,01-2,50$ & Emergente & Superficial \\
\hline Scirpus californicus (C.A. Mey.) Steud. * & $0,01-4,12$ & Emergente & Superficial \\
\hline Callitriche heterophylla Pursh & $0,01-0,40$ & Sumergida & Superficial \\
\hline Elodea canadensis Rich. * & $0,40-4,10$ & Sumergida & Medio \\
\hline Myriophyllum quitense Kunth & $0,40-5,50$ & Sumergida & Medio \\
\hline Potamogeton striatus Ruiz \& Pav. & $0,40-5,70$ & Sumergida & Medio \\
\hline Ceratophyllum demersum L. & $1,00-7,00$ & Sumergida & Profundo \\
\hline Potamogeton pectinatus L. & $1,80-5,50$ & Sumergida & Profundo \\
\hline Potamogeton illinoensis Morong & $1,80-6,00$ & Sumergida & Profundo \\
\hline \multicolumn{4}{|l|}{ Especies de Yahuarcocha } \\
\hline Lemna minuta Kunth & $0,00-0,00$ & Flotante & Superficial \\
\hline Eichhornia crassipes (Mart.) Solms. & $0,00-0,00$ & Flotante & Superficial \\
\hline Azolla filiculoides Lam. & $0,00-0,00$ & Flotante & Superficial \\
\hline Myriophyllum aquaticum (Vell.) Verdc. & $0,00-0,50$ & Flotante & Superficial \\
\hline Bacopa monnieri (L.) Wettst & $0,01-0,10$ & Emergente & Superficial \\
\hline Scirpus californicus (C.A. Mey.) Steud. & $0,01-2,50$ & Emergente & Superficial \\
\hline Typha domingensis Pers. & $0,01-2,50$ & Emergente & Superficial \\
\hline Elodea canadensis Rich. & $0,20-2,00$ & Sumergida & Medio \\
\hline Potamogeton pusillus L. & $1,90-3,10$ & Sumergida & Profundo \\
\hline
\end{tabular}

\section{* Especies compartidas}


Tabla 3. Valores promedio de la concentración de macronutrientes tomados en cada transecto y que inciden en la productividad de los lagos de San Pablo y Yahuarcocha

\begin{tabular}{|c|c|c|c|c|c|c|c|c|}
\hline & Ca mg/l & Mg mg/l & $\mathrm{Na} \mathrm{mg} / \mathrm{l}$ & Fe mg/l & $\mathrm{Pb} \mathrm{mg} / \mathrm{l}$ & Al mg/l & $\mathrm{SO}_{4} \mathrm{mg} / \mathrm{l}$ & $\mathrm{K} \mathrm{mg} / \mathbf{l}$ \\
\hline San Pablo & 0.51 & 17.0 & 31.9 & 3.72 & 0.00 & 0.53 & 2.18 & 3.38 \\
\hline Yahuarcocha & 6.73 & 49.5 & 82.8 & 0.89 & 0.04 & 0.40 & 1.18 & 20.20 \\
\hline
\end{tabular}

Por su parte, El pH y la temperatura del agua fueron los parámetros de mayor variación entre transectos, dentro de una misma laguna, mientras que los valores de conductividad y alcalinidad variaron entre las dos lagunas (Tabla 4). El pH y la temperatura del agua muestran una correlación positiva entre sí(Spearman $=0.79, \mathrm{p}<0.05)$, aligual que el $\mathrm{pH}$ y la transparencia (Spearman $=0.64$, $\mathrm{p}<0.05)$ y en menor grado entre la temperatura $\mathrm{y}$ la conductividad (Spearman $=0.48, \mathrm{p}<0.05$ ).

Tabla 4. Parámetros físico-químicos tomados en cinco transectos (análisis "in situ”) a diferentes profundidades en las lagunas de San Pablo y Yahuarcocha.

\begin{tabular}{|c|c|c|c|c|c|c|}
\hline & $\begin{array}{l}\text { Profundidad } \\
\text { (m) }\end{array}$ & $\begin{array}{l}\text { Conductividad } \\
\text { (ms) }\end{array}$ & $\mathrm{pH}$ & $\begin{array}{l}\text { Transparencia } \\
\text { Secchi (m) }\end{array}$ & $\begin{array}{l}\text { Temperatura } \\
\left({ }^{\circ} \mathrm{C}\right)\end{array}$ & $\begin{array}{l}\text { Alcalinidad } \\
(\mathrm{mg} / \mathrm{l})\end{array}$ \\
\hline \multicolumn{7}{|c|}{ San Pablo } \\
\hline \multirow[t]{3}{*}{$\mathrm{T} 1$} & 8.10 & 300 & 7.4 & 3.10 & 19.3 & 226 \\
\hline & 4.10 & 310 & 6.8 & 3.10 & 19.5 & 226 \\
\hline & 0.50 & 310 & 7.7 & 3.10 & 19.8 & 226 \\
\hline \multirow[t]{3}{*}{ Т 2} & 6.00 & 310 & 7.0 & 3.50 & 18.4 & 226 \\
\hline & 3.00 & 310 & 7.1 & 3.50 & 18.4 & 226 \\
\hline & 0.50 & 300 & 7.4 & 3.50 & 18.6 & 226 \\
\hline \multirow[t]{3}{*}{ T 3} & 6.00 & 320 & 6.2 & 3.00 & 17.3 & 226 \\
\hline & 3.00 & 310 & 6.7 & 3.00 & 17.4 & 226 \\
\hline & 0.50 & 320 & 6.8 & 3.00 & 17.5 & 226 \\
\hline \multicolumn{7}{|c|}{ Yahuarcocha } \\
\hline \multirow[t]{2}{*}{ T 1} & 4.00 & 680 & 8.2 & 1.60 & 22.1 & 956 \\
\hline & 2.00 & 680 & 8.5 & 1.60 & 21.8 & 956 \\
\hline \multirow[t]{2}{*}{ T 2} & 3.00 & 720 & 7.9 & 1.20 & 21.2 & 956 \\
\hline & 1.00 & 620 & 8.2 & 1.20 & 21.0 & 956 \\
\hline
\end{tabular}

\section{DISCUSIÓN}

\section{Riqueza de especies florísticas.-}

En un ecosistema acuático la riqueza de especies está determinada por la heterogeneidad de hábitat. En las lagunas estudiadas esta riqueza es mayor en zonas palustres, donde la vegetación emergente está dominada por monocotiledóneas, que además de la reproducción sexual (flores) tienen reproducción vegetativa (estolones y rizomas), comoadaptación haciala vida acuática. Esto les permite ser más competitivas cuando la sedimentación y los cambios en el nivel del agua son drásticos (Hutchinson, 1975; Sculthorpe, 
1967). La dominancia de monocotiledóneas rizomatosas en zonas palustres contribuye a la sedimentación y desecación de las lagunas por los altos niveles de evapotranspiración (Arrignon, 1979). Consecuentemente, se esperaría que estas especies ganen espacio y las lagunas sufran transformaciones drásticas con el tiempo, condenándolas a su desaparición (Roldán y Ramírez, 2008).

La riqueza de especies en los ecosistemas acuáticos está determinada por el grado de eutrofización de los cuerpos de agua (Wijninga et al., 1989; Chambers y Kalff, 1985); por tanto, ecosistemas con moderada concentración de nutrientes (mesotróficos), como la laguna San Pablo, presentan mayor riqueza de especies pero menor dominancia que los ecosistemas ricos en nutrientes (eutróficos) como Yahuarcocha donde P. pusillus se ha transformado en una especie invasiva en toda la laguna. Además, San Pablo tiene mayor riqueza de especies debido a su extensión y heterogeneidad de hábitat, lo cual crea condiciones óptimas para el establecimiento de muchas especies con diferentes formas de vida (Rojas y Novelo, 1995). Por ejemplo, C. demersum, es la única especie sumergida que carece de un sistema radical verdadero (Raven et al., 1999), lo cual le permite dispersarse con facilidad para colonizar espacios vacíos. Esta especie puede presentar dependencia frente a algún componente del agua o sustrato aún desconocido, pero que podría relacionarse con concentraciones elevadas de sales de azufre (Tabla 3), por la influencia volcánica que tiene este cuerpo de agua, y no únicamente por la presencia de materia orgánica como lo manifiesta Kiersch et al. (2004). Además su presencia es exclusiva para la laguna de San Pablo, lo cual que lleva a pensar que existe una interacción de dependencia muy cercana con el Bass (Morone saxatilis), especie de pez también registrada exclusivamente para esta laguna en el Ecuador.

\section{Estratificación de las especies.-}

Existe una elevada correlación entre la transparencia del agua y la estratificación de la vegetación. A mayor transparencia, mayor estratificación (Middelboe y Markager, 1997; Duarte y Kalff, 1987; Chambers y Kalff, 1985). Este fenómeno tiene ventajas frente a la ubicación latitudinal de Ecuador, ya que por cada grado de incremento en latitud, el requerimiento de luz aumenta en $0.04 \%$ (Middelboe y Markager, 1997), lo cual permite que las plantas acuáticas colonicen a mayores profundidades en los países asentados sobre la línea ecuatorial.

Las lagunas San Pablo y Yahuarcocha están dominadas por angiospermas caulescentes como Myriophyllum, Ceratophyllum y Potamogeton, las mismas que se encuentran creciendo en profundidades superiores a la lectura de disco Secchi (Tabla 2). Lo cual demuestra que estas especies están adaptadas a sobrevivir bajo condiciones limitadas de luminosidad. En Ecuador la incidencia de luz es homogénea, constante y más intensa que en países ubicados a menor o mayor latitud, por tanto la profundidad de colonización aumenta en $0.12 \mathrm{~m}$ por cada grado latitudinal, favoreciendo el establecimiento de plantas acuáticas a mayores profundidades (Middelboe y Markager, 1997).

\section{La química del agua en la distribución de las especies.-}

La alcalinidad se utilizó como uno de los parámetros para medir el estado trófico de las lagunas, ya que esta refleja la cantidad de iones bicarbonato y carbonato presentes en el agua, los cuales permiten que los procesos biológicos de respiración y fotosíntesis se lleven a cabo (Roldán, 1995). La alcalinidad depende en gran parte de la naturaleza del terreno. En las lagunas de San Pablo y Yahuarcocha los niveles de alcalinidad superan los $200 \mathrm{mg} / \mathrm{l}$. En contraste, lagos de zonas intermedias y de los páramos registran alcalinidades entre 10 y $80 \mathrm{mg} / 1$ y las ciénagas y lagunas costeras registran valores superiores a los $100 \mathrm{mg} / 1$ (Roldán, 1995). La alcalinidad (926 mg/l) en Yahuarcocha ( $\mathrm{pH}$ 8) demuestra que el carbono disuelto en el agua se encuentra disponible en forma de carbonatos, lo cual no es aprovechable en su totalidad por las plantas (Wetzel, 1960), y por lo tanto se transforma en elemento contaminante para el ecosistema. 
Otro factor que ayuda a determinar el estado trófico es la conductividad, cuyos valores en las lagunas de San Pablo y Yahuarcocha (>300 $\mathrm{mS} / \mathrm{cm}$ ) indican mayor concentración de iones disueltos en el agua (i.e., $\mathrm{Ca}, \mathrm{Na}, \mathrm{K}, \mathrm{Mg}$, $\mathrm{Fe}, \mathrm{Pb}, \mathrm{Al}$, $\mathrm{SO}_{4}$ ). En los trópicos los valores de conductividad están relacionados con la naturaleza química del terreno (Roldán, 1995). En San Pablo y Yahuarcocha las conductividades son mucho más altas por la acumulación de materiales de desecho arrastrados por los afluentes que lo alimentan (Carrera de la Torre, 1990). El Ca y el $\mathrm{Mg}$, además de indicar el grado de dureza de las aguas, son los minerales que más aportan en la productividad primaria de los ecosistemas (Wetzel, 2001; Goldman y Horne, 1968). El Ca es importante para la formación de huevos y estructuras óseas en los organismos acuáticos. El Mg constituye el núcleo de la molécula de clorofila (Roldán, 1995). En las dos lagunas estudiadas se registran valores representativos de Ca y Mg (Tabla 3), lo cual crea condiciones óptimas para el desarrollo de ciertas especies de peces comestibles como el Bass (Morone saxatilis), la Carpa (Cyprinus carpio), la Tilapia (Tilapia mozambique) y otros peces ornamentales como los Guppis (Lebistes reticulatus) y los Espadas (Xiphophorus helleri) (Bailey y Sandford, 2005).

Los sulfatos disueltos en agua favorecen la incorporación de ciertas proteínas en los organismos y contribuyen a la proliferación de algas verdes. Por esto, Yahuarcocha es un lago de coloración verde intenso. En zonas donde existe un volcán, como a las orillas de la laguna San Pablo, también es común encontrar grandes concentraciones de $\mathrm{SO}_{4}$, por la composición geológica natural del sitio donde se asienta el cuerpo de agua.

El hierro es otro elemento que se encuentra en altas concentraciones en San Pablo. Esto puede provocar que los estratos profundos $(>10 \mathrm{~m})$ tengan poco oxígeno, e impedir la vida de organismos superiores como plantas acuáticas y peces; además, evita que los procesos de descomposición de la materia se lleven a cabo rápidamente (Roldán, 1995). La presencia de plomo en Yahuarcocha podría ser un indicador de la influencia de la pista automovilística asentada en los alrededores de la laguna (Carrera de la Torre, 1990).

En conclusión, de acuerdo con los niveles tróficos propuestos por Roelofs y Bloemendaal (1988), la laguna de San Pablo es un ecosistema mesotrófico que en un futuro cercano puede convertirse en eutrófico como Yahuarcocha. Se puede predecir que las poblaciones naturales de plantas y peces como C. demersum y Bass (Morone saxatilis), especies que se encuentran únicamente en el lago San Pablo y en ninguna otra parte de Ecuador, corren el riesgo de poder desaparecer.

La gran extensión del lago San Pablo (583 ha) y su diversidad de microhábitat permiten que la riqueza florística sea mayor a la de Yahuarcocha. Sin embargo, los grandes asentamientos humanos, las actividades agrícolas y ganaderas y el uso indiscriminado de los recursos, alteran el estado trófico de la laguna San Pablo a un ritmo acelerado y es probable que su riqueza de especies se reduzca a los niveles de la de Yahuarcocha.

\section{AGRADECIMIENTOS}

Agradezco al proyecto ENRECA (Enhansement of Research Capacity) de Dinamarca, que en colaboración conla PUCE (Pontificia Universidad Católica del Ecuador) financiaron este proyecto de investigación. Varias personas contribuyeron en su ejecución, y en especial dejo constancia de mi agradecimiento al señor Luis Eriberto Luna, quien colaboró eficientemente en el trabajo de campo; a Alex Fraser de la Universidad de Plymouth, Inglaterra, por los análisis químicos de las muestras de agua; a Dean Jacobsen por sus valiosas sugerencias en la elaboración del manuscrito; a Henrik Balslev y Renato Valencia por su colaboración en la revisión del artículo.

\section{REFERENCIAS BIBLIOGRÁFICAS}

Arrignon J. 1979. Ecología y piscicultura de aguas dulces. Mundi-Prensa, Madrid, España.

Bailey M. y Sandford G. 2005. The new guide to aquarium fishes: A comprehensive and 
authoritative guide to tropical freshwater, brackish, and marine fishes.

Casallas J y Gunkel G. 2002. Algunos aspectos limnológicos de un lago altoandino: El Lago San Pablo, Ecuador. Limnetica 20 (2), 29-46

Carrera de la Torre L. 1990. Estudio para la recuperación y protección de la zona turística de los lagos de la provincia de Imbabura con fines de equilibrio ecológico y promoción turística. (Informe general (Diagnóstico y Propuesta), Ministerio de Relaciones Exteriores, Ecuador.

CEPCU. 1999. Boletín del Centro de Estudios Pluriculturales. Rev Imbacocha, Otavalo, Ecuador, vol. 7-8.

Chambers P y Kalff J. 1985. Depth distribution and biomass of submersed aquatic macrophyte communities in relation to secchi depth. Can J Fish Aquat Sci 42: 701-709.

Cortés A, Mendivil R, Cuevas R y García C. 1980. Algunos aspectos físicos y químicos y consideraciones sobre la pesca en el lago de Cuitzeo, Mich. (Estudio trimestral 19761977). En: Memorias del Segundo Simposio Latinoamericano de Acuacultura. Departamento de Pesca. México, DF 3: 1979-2020.

Duarte C. y Kalff J. 1987. Latitudinal influences on the depth of maximum colonization and maximum biomass of submersed angiosperms in lakes. Can J Fish Aquat Sci 44: 1759-1764.

Goldman CR. y Horne A. 1968. Limnology: origin of lakes and estuaries, eutrophycation and paleolimnology 18: 341-360.

Gunkel G. 2000. Limnology of an equatorial high mountain lake in Ecuador, Lago San Pablo. Limnologica 30: 113-120.
Hutchinson G. 1975. A treatise on limnology. Vol. III. Limnology botany. John Wiley and Sons. Nueva York.

Kent M y Coker P. 1992. Vegetation: description and analysis, a practical approach. Belhaven Press, Londres, pp. 112-161.

Kiersch B, Mühleck R y Gunkel G. 2004. Las macrófitas de algunos lagos altoandinos del Ecuador y su posible utilización como bioindicadores. Rev. Biol. Trop. 51(4)

Mallin M. 2000. Impact of industrial animal production on rivers and estuaries. En: American Scientist, The magazine of sigma XI, The Scientific Research Society 88: 26-37.

Middelboe A y Markager S. 1997. Depth limit and minimum light requirements of fresh water macrophytes. Freshwat Biol 37: 553-568.

Mueses TE. 2010. Determinación de la dinámica poblacional de avifauna en la laguna de Yahuarcocha y propuesta de estrategias de conservación. Tesis de Ingeniería, Escuela de Ingeniería en Recursos Naturales Renovables. Universidad Técnica del Norte. Imbabura, Ecuador.

Norman G y Streiner D. 1998. Bioestadística. Mc.Master University, Ontario-Canadá.

Raven P, Evert R y Eichhorn S.1999. Biology of plants. Sixth ed. Freeman and Company Worth Publishers.

Rojas J y Novelo A. 1995. Flora y vegetación acuática del lago de Cuitzeo, Michoacán, México. Acta Bot Mex 31: 1-17.

Roelofs J y Bloemendaal F. 1988. Trofie. En: Bloemendaal and Roelofs (eds.). Waterplanten en waterkwalitei. Koninklijke Nederlandse Nattuurhistorisch Vereninging. Utrecht. nr., pp. 113-125. 
Roldán G. 1995. Fundamentos de limnología neotropical. Universidad de Atioquia. Pp. 251-267.

Roldán G y Ramírez J. 2008. Fundamentos de limnología Neotropical, 2da. Edición. Editorial Universidad de Antioquia. Medellín, Colombia. Pp. 440.

Sculthorpe CD. 1967. The biology of aquatic vascular plants. New York: St. Martin's Press.

Terneus E. 2002. Estructura y Composición florística de las plantas acuáticas en 70 lagunas altoandinas del Ecuador. Tesis doctoral. Escuela de Ciencias Biológicas, Pontificia Universidad Católica del Ecuador., Quito, Ecuador.
Terneus E. 2001. Aquatic plant communities of the páramo lakes of volcán Chiles, Ecuador. En: P.M Ramsay (ed.). The ecology of volcán Chiles: high- altitude ecosystems of the Ecuador-Colombia border. Plymouth: Pebble \& Shell. Pp. 55-63.

Wetzel R. 2001. Limnology: lake and river ecosystems. San Diego, Academic press.

Wetzel R. 1960. Metabolismo gaseoso de los macrófitos acuáticos. En:Limnología. 1981 Omega SA. (eds.). pp. 135-152. Barcelona-España.

Wijninga V, Rangel O y Cleef A. 1989. Botanical ecology and conservation of the laguna de La Herrera (Sabana de Bogotá, Colombia). Caldasia 16 (76):23-40. 